\title{
REFERENCIAS
}

HACKING, Ian (1983); Reprasenting and Intervening, Cambridge, Cambridge University Press.

- (1992): aThe Self-Vindication of the Laboratory Sciencess, en A. Pickering (ed.), Science as Practice atd Culute, Chicago, The University of Chicago Press, 29-64.

Hickman, Iany (1990): John Dewey's Pragnatic Tech tology, Bloomington, Indiana University Press.

IHDE, Don (1991): Instrumental Realism. The Interface between Philosophy of Science and Philosophy of Technology, Bloomington, Indiana University Press.

JANich, P. (1978): "Physics - Natural Science or Technology?*, en W. Krohn, E. Layton $y$ P. Weingart (eds.). The Dynamics of Science and Technology, Dordrecht, Reidel, 3-27.

- (1988): :Truth as Success of Action. The Constructive Approach in the Philosophy of Sciencex, en K. Hronszky, M. Feher y B. Dajka (eds.). Scientific Knowledge Socialized, Dordrecht, Kluver, 313-326.

LAtour, Buno (1994): «On Technical Medition Philosophy, Sociology, Genealogym, Conmon Knowledge, 29-63.

LORENZEN, Paul (1978): Theorie der technischen und politischen Vernunf, Stutgart, Reclam.
- (1986): «Das technische Fundament der Geometrie; en $C$. Bumichter, $R$. Inhetveen y $R$. Kotter (eds.). Techntische Rationalität und rationale Heuristik, Munich, Schoningh, 17-25.

- (1987): Lehrbuch der konstruktiven! Wissenschaftsheorie, Mannheim, Wissenschaftsverlag.

MiтchaM, C. (1994); Thinking through Technology. The Path between Engineering and Philosophy, Chicago, The University of Chicago Press.

Prckerunc, A. (1992): «From Scjence as Knowledge to Science as Practicex, en A. Pickering (cd.), Science as Practice and Culture, Chicago, The University of Chicago Press, 1-26.

- (1993): «The Mangle of Practice: Agency and Emergence in the Sociology of Science, Anterican Jountal of Sociology, 99, n." 3, 559-589.

Puce, Derek Desolla (1984): "The Seience/Technology Relationship, the Craft of Experimental Science, and Policy for Improverment of High Technology Innovation, Research Policy, $13,3-20$

TETENS, Holm (1987): Experinentelle Erfahruny. Eine wisseschafstheoretische Studie iber die Rolle des Experiments in der Begriffs- und Theoriebilany der Physik, Hamburgo, Felix Meiner.

\section{La sociología y la naturaleza social de la ciencia}

\author{
JESÚS SÁNCHEZ NAVARRO \\ Universidad de La Laguna
}

En 1928, en pleno auge del Positivismo Lógico, Carnap propuso un programa de trabajo que tuvo importantes consecuencias posteriores. Según Carnap la Epistemología debía realizar una reconstrucción racional de los procesos de conocimiento y 'conformación de la realidad' que en la mayoría de los casos llevamos a cabo intuitivamente. Consideraba tal reconstrucción descriptiva, fidedigna y siguiendo ula forma racional de derivaciones lógicas». Llevarla a cabo con los conceptos de todos los campos del conocimiento, incluyendo la ciencia, era desde su punto de vista el problema fundamental de la filosotia y la epistemología."

Diez años después y aplicando la propuesta de Carnap a la Filosofía de la Ciencia, Reichenbach establecia dos distinciones que ya habian sido insinuadas por otros autores y que hicieron fortuna rápidamente. ${ }^{2}$ La primera era la diferencia entre las relaciones internas y externas del conocimiento. Llamaba 'internas' a las que 
se dan entre los contenidos del conocimiento y deben realizarse de hecho para poder comprenderlo, $y$ 'externas' a las que combinan el conocimiento con otros factores que no pertenecen a su contenido. $\mathbf{E}$ inmediatamente añadía: "la epistemología está interesada sólo en las relaciones internas, mientras que la sociología, aunque puede considerar parcialmente relaciones internas, siempre las mezcla con relaciones externas [...] Podemos entonces decir que a la tarea descriptiva de la epistemología le interesa la estructura interna del conocimiento y no las características externas que se le presentan a un observador que no toma en consideración su contenidow. ${ }^{3}$ La segunda distinción era entre contexto de descubrimiento y contexto de justificacion. Al primero correspondería el conjunto de procesos dc pensamiento $\mathrm{y}$ operaciones psicológicas que ocurren de hecho, incluyendo los de convicción y aceptación subjetiva. Al segundo, las reconstrucciones racionales, en sentido camapiano, de esos procesos. El propio Reichenbach ilustra el primer contexto como la forma en que los procesos de pensamiento o los descubrimientos se llevan a cabo subjetivamente, y el segundo como la forma en que son presentados en público o justificados lógicamente. Para el, sólo el contexto de justificación interesa a la epistemologia, porque lo que ésta busca es "construir los procesos de pensamiento en una forma en que estos deben ocurrir si han de situarse en un sistema consistente; o construir conjuntos justificables de operaciones que puedan ser intercalados entre el punto de partida y los procesos de pensamiento resultantes, reemplazando los enlaces intermedios reales. Así, la epistemolo. gía considera un sustituto lógico más que procesos reales\%. ${ }^{4}$

\section{La concepción clásica de la ciencia}

Puesto que la ciencia se consideraba la forma más perfecta de conocimiento, estos planteamientos se le aplicaban con toda precision $y$, al combinarse con el criterio de demarcación, llevaban a considerar el conocimiento cientffico dotado de cuatro propiedades fundamentales: las de intersubjetividad, decidibilidad empírica, racionalidad y objetividad, a las que se añadiría, como consecuencia inmediata, la de neutralidad cognitiva. Aunque estas características eran más propias de las recontrucciones racionales y sus idealizaciones consiguientes que de los procesos científicos reales, acabaron siendo identificadas como sus características distintivas. ${ }^{5}$ El desarrollo posterior de la filosofía de la ciencia, unido a críticas tan influyentes como las de Popper, debilitaron la noción fuerte de reconstrucción racional limitando su alcance a las «contrastaciones subsiguientes mediante las que se puede descubrir que cierta inspiración fue un descubrimiento a se puede reconocer como un conocimiento" " en lugar de aplicarse al proceso de conocimiento completo. De la misma forma, las dos distinciones de Reichenbach se mezclaron, pasando a integrarse los factores externos en el contexto de descubrimiento, aunque ambos contextos continuaron separados tajantemente, considerándose que los procesos del primero no influían en los del segundo. Pero esto no afectó a la imagen de la ciencia, que se mantuvo caracterizada por las propiedades antes citadas, ni a la naturaleza descarnada de la reconstrucción, que seguía siendo sin sujeto cognoscente o referida a científicos idealmente racionales. I as desviaciones observadas en la práctica real de individuos o grupos serían achacables a limitaciones en su racionalidad 
o a la intervención de factores externos o internos no lógicos y sólo sería lícito recurrir a ellos para dar cuenta de estas desviaciones. ${ }^{7}$

De esta forma no sólo se establece una distinción tajante entre la ciencia como institución social y la ciencia como conocimiento considerado objetivo, sino que también se elimina a los sujetos al considerar el conocimiento científico (sólo se recurre a ellos para explicar el error o la desviación flagrante respecto a los procesos reales). Igualmente, se acaba identificando la ciencia con su resultado último, es decir, conocimiento público lógicamente justificado, y la actividad cientifica con sus productos finales, las teorías cientificas, ambos claramente diferenciados de sus procesos de producción y de los diversos factores implicados en ellos, que no afectan o no deberían afectar a los contenidos del conocimiento resultan. te. $Y$, finalmente, se establece una división del trabajo clara y precisa entre la epistemología y la filosofía de la ciencia, por una parte, y la sociología o la psicologra de la ciencia, por otra.

\section{Soctologia de la ciencia y sociologta del conocimiento}

Los mismos sociólogos aceptaron durante mucho tiempo estas tesis y la división del trabajo resultante. Así, la sociologia de la ciencia mertoniana centraba su atención en los aspectos institucionales de la ciencia, desde las condiciones externas que favorecen su constitución y desarrollo como institución hasta su legitimación y la evaluación social de los descubrimientos cientificos, pero sin entrar en su contenido cognitivo..$^{\sharp}$ Lo que le interesaba eran las normas y organización de la ciencia como institución social, sus relaciones con otras instituciones y su integración o desintegración en la es- tructura social. En última instancia, su propia concepción de la sociología de la ciencia como una disciplina empirica y 'cientifica', capaz de usar indicadores cuantitativos en lugar de análisis cualitativos y que no mezclara niveles distintos, es decir, que sólo hiciera descripciones sociológicas y las explicara y fundamentara socialmente, llevaba a Merton a poner la atención en la estructura social de la ciencia más que en la producción del conocimiento." En el plano opuesto a la sociología de la ciencia de Merton se situaba la sociología del conocimiento de Mannheim. Básicamente cualitativa, sintética, casi omnicomprensiva, basada en el análisis ideológico, conscientemente valorativa e incluso dotada de un componente normativo y con asunciones teóricas y filosóficas tan profundas que llegaba a la confrontación explícita con la epistemología, la sociología del conocimiento defendía la influencia determinante de los factores sociológicos e ideológicos sobre los contenidos cognitivos, su justificación e incluso la organización de la experiencia. ${ }^{10} \mathrm{Sin}$ embargo, la ciencia natural (a la que Mannheim llama formal, exacta, etc.) quedaba fuera de este marco: su contenido cognitivo, su validez y justificación no serían afectados, en general, por los factores externos. En palabras de Mannheim: «Puede decirse del conocimiento formal que es esencialmente accesible a todos y que su contenido no es afectado por los sujetos individuales y sus afiliaciones histórico-sociales". "Las razones para otorgar a la ciencia natural este estatus diferenciado dentro del conocimiento humano son diversas: la universalidad de sus afirmaciones y aplicaciones, el recurso a demostraciones y prucbas, sus componentes cuantitativos y formales, la homogeneidad de los problernas que enfrenta, la búsqueda de intersubjetivi- 
dad y neutralidad tanto en la experiencia utilizada, como en sus criterios de verdad, etc., ${ }^{12}$ pero, como puede apreciarse, todas ellas son 'internas' y se basan, en última instancia, en la aceptación de las características asignadas a la ciencia por el modelo positivista.

\section{La sociologta del conocimiento cientifico}

Precisamente, el desarrollo de la Sociología del Conocimiento Científico a partir de los años setenta está estrechamente conectado con la crisis de esa concepción de la ciencia. Las criticas de Sellars, Quine, Putnam, Rorty, etc., desde cl punto de vista de la Epistemología y de Hanson, Toulmin, Feyerabend, Kuhn, etc., desde la Filosofía de la Ciencia pusieron en tela de juicio la validez de la distinción entre contextos, las reconstrucciones racionales e incluso las características de objetividad, intersubjetividad, decidibiJidad, racionalidad y neutralidad cognitiva $o$, al menos, la forma en que tradicionalmente se habian definido, lo que permitio abrir la 'caja negra' de la ciencia, su contenido cognitivo, a los análisis sociológicos. ${ }^{13}$ En términos generales, se mantenía que las distinciones clásicas de Carnap, Reichenbach, Popper, etc., eran artificiales y conducían a una imagen de la ciencia que poco tenía que ver con la real. De la misma manera, se mantenía que el conocimiento científico no necesita una fundamentación previa más allá de su propia eficacia, por lo que lo impor* tante es dar cuenta de su naturaleza y desarrollo reales, no reconstruirlo racionalmente. En consecuencia, los sociologos del conocimicnto cientifico rechazan las reconstrucciones racionales, la identificación de la ciencia con sus resultados, la separación entre los aspectos institucionales y cognitivos de la ciencia y cualquier otra limitación impuesta al estudio sociológico del conocimiento cientifico. Por el contrario, defienden que la ciencia es una actividad esencialmente social y que es imprescindible tomar en cuenta a los sujetos actores y su comportamiento real.

En cierto modo, es como si se hubiera producido una combinación entre la sociologia del conocimiento de Mannheim y la sociología de la ciencia de Merton, de ahi el nombre de Sociologfa del Conocimiento Científico, aunque institucionalmente se les denomina Estudios Sociales de la Ciencia. Pese a que dentro de estos estudios hay diversas corrientes, la afirmación fundamental de todas ellas es que los factores sociales condicionan, e incluso determinan, los contenidos y la aceptación del conocimiento científico y que tanto éstos como las características fundamentales de la ciencia son resultado de la interacción social y no su condición previa. En este sentido, es posible establecer cinco principiosguía compartidos en mayor o menor medida por todas ellas: ${ }^{14}$

1. El principio de Relativismo. Se niega que existan criterios absolutos y fundacionales que garanticen la verdad o la racionalidad. Aunque los juicios y decisiones de los cientificos se reclamen racionales y sus afirmaciones pretendan ser verdaderas, tanto la noción de verdad, como las de progreso y racionalidad, son revisables y relativas a comunidades, épocas y contextos concretos. También las normas y valores que guían la actividad cientifica son cambiantes y relativos, pues son producto de procesos sociales dentro de la comunidad científica. Así, la producción, el desarrollo y el cambio del conocimiento científico no son procesos autónomos, ni objetivos, sino resultado de negociaciones y procesos de inter- 
acción social entre científicos. Lo que se entienda por ciencia, su validez y aceptabilidad, al igual que los métodos utilizados, son cuestiones relativas. Como puede observarse, este principio choca con el supuesto de objetividad.

2. El principio de Constructivismo. Las representaciones científicas no provienen directamente de la realidad, ni son un reflejo literal de ésta. No puede esperarse siquiera una interpretación idéntica de los mismos fragmentos de evidencia, pues la experiencia no es neutral sino dependiente y varía según el contexto, los aprendizajes, los esquemas compartidos y los procesos de comunicación en que se produzca. De ahí que el conocimiento $y$, en cierto modo, la realidad se consideren socialmente construidos. El supuesto afectado en este caso es el de intersubjetividad.

3. El principio de Instrumentalismo. E] conocimiento científico no difiere sustancialmente de otros conocimientos, salvo quizá por su mayor eficacia y efectividad. De ahí que posea una función instrumental y pragmática, puesto que pretende conseguir ciertos fines o satisfacer ciertos intereses, fines o intereses que están, en última instancia, socialmente determinados; por esta razon, su producción y aceptación están fúertemente condicionadas por su capacidad para cumplir esa función. Su antagonista clásico es el supuesto de decidibilidad empírica.

4. El principio de Naturalización. Rechazan la separación entre los procesos de descubrimiento y los de justificacion y validación del conocimiento resultante. Muy al contrario, los primeros, y con ellos los factores sociológicos, psicologicos, etc., influyen en los segundos e incluso en la forma en que se organiza el conocimiento científico. De ahí que el estudio empírico-social de los procesos de producción del conocimiento científico y de los procesos de formación de creencias sea fundamental para el estudio de la ciencia. Tanto los procesos de producción del conocimiento científico, como los contenidos resultantes y su evaluación, son considerados fenómenos naturales y susceptibles de investigación empirica. En el caso que nos ocupa, esta naturalización se entiende como sociologización: la investigación empírica ha de ser fundamentalmente sociológica, porque el componente social es previo $\mathrm{y}$ más fundamental que cualquier otro. De ahí que la racionalidad no sea algo previamente dado, ni un ideal regulativo, sino que se constituye en los procesos de interacción social.

5. El principio de Causación Social. La actividad científica no es llevada a cabo por sujetos epistémicos ideales, sino por comunidades concretas organizadas socialmente, que a su vez están inmersas en estructuras sociales más amplias. En este sentido los cientificos son criaturas humanas y sociales sujetas a los mismos tipas de explicación que cualquier otro grupo. Y el conocimiento que producen es en buena medida resultado y reflejo de la forma en que se organizan e interactúan dentro de esas comunidades y de las estructuras sociales generales en que esas comunidades se sitúan. Es el principio distintivo de la Sociologia del Conocimiento Científico y choca frontalmente con el supuesto de la neutralidad cognitiva y con cualquier proyecto de reconstrucción racional.

Como puede apreciarse, los cuatro primeros principios son más filosóficos que sociológicos y podrían encontrarse formulaciones parecidas en diferentes epistemólogos y filósofos de la ciencia, el quinto es el que produce el sesgo radicalmente sociologico, y la combinación de los cinco lo que convierte a la Sociología del Conocimiento Científico en una propuesta tan provo- 
cativa. Igualmente, se observa que estos planteamientos no conducen tanto a la negación radical de la objetividad, la intersubjetividad, la decidibilidad y la racionalidad, cuanto a su redefinición desde un punto de vista sociológico: todas ellas serían construidas en la interacción social y la práctica real, histórica o cotidiana, de los actores y grupos involucrados en la actividad científica. ${ }^{15}$ Por eso, cualquier intento de fundamentar el conocimiento científico ha de ser esencialmente sociológico. No obstante, dentro de la Sociología del Conocimiento Científico existen numerosas variantes que difieren cntre $s_{1}$, e incluso llegan a rechazarse mutuamente. ${ }^{16}$ Por razones de espacio nos limitaremos a señalar brevemente algunas de las características esenciales de las tres más conocidas: el Strong Programme, el Programa Relativista y la Etnografía de la Ciencia.

\section{El Strong Programme}

El Strong Programme o Programa Fuerte de Sociologia do la Ciencia es la propuesta fundacional de la Sociologia del Conocimiento Cientffico y fue desarrollada a comienzos de los años setenta por Barnes, Bloor y la Escuela de Edimburgo (MacKenzie, Shapin, etc.). ${ }^{17}$ Como su nombre indica, no sólo es un programa para el estudio social de la ciencia, sino también para la redefinición y constitución de la sociología de la ciencia como disciplina científica. Esta pretensión condiciona de forma decisiva sus planteamientos, pues propone construir una sociología de la ciencia que siga el modelo de la ciencia 'dura': dar explicaciones causales a partir de leyes sociales empíricamente comprobables. ${ }^{18}$ Por eso, aun aceptando los cinco principios antes citados, pone especial énfasis en la naturalización y la causación social y se centra básicamente en las relaciones de la ciencia con la sociedad general en que se produce. Por ello, también, analiza fundamentalmente casos históricos para establecer cómo la sociedad influye en la producción y aceptación de la ciencia. Esta influencia tiene lugar en un triple sentido: 1) Lo que se considere ciencia en un momento determinado está mediatizado por la sociedad en que se genera. A fin de cuentas lo que se entienda por ciencia será lo que la comunidad científica considere como tal y la comunidad cientifica no es más que un subgrupo social especificamente organizado dentro de la sociedad y reconocido por ésta. 2) La actividad cientifica y el conocimiento resultante son productos del trabajo de los individuos en el seno de la comunidad científica. Por tanto, la estructura de esa comunidad, su organizacion y sus relaciones internas determinarán su forma y naturaleza. 3) La actividad cientifica ha sido siempre socialmente reconocida $y$, al menos desde el siglo XIX, está profesionalizada. Eso hace que los factores macrosociales externos influyan en la forma y el funcionamiento de la comunidad, en sus disputas y acuerdos y en los resultados obtenidos.

A su vez, sus objetivos fundamentales serían, primero, describir cómo (y si es posible por que) en épocas distintas grupos sociales distintos seleccionan diferentes aspectos de la realidad como objeto de estudio y explicación científica $y_{2}$ segundo, describir cómo se construyen socialmente la observación, los experimentos, la interpretación de los datos y las propias creencias científicas en la doble vertiente de construcción por parte de la comunidad y construcción por parte de la sociedad. ${ }^{19}$ Para conseguirlo parten de cuatro postulados básicos: 1) el postulado de imparcialidad respecto a lo 
que se considera en una epoca determinada conocimiento verdadero o falso, racional o irracional; ninguna de estas dicotomias ha de darse por supuesta, sino que requiere explicación; 2) el postulado de simetría, por el cual todo el conocimiento, con independencia de su éxito o fracaso, ha de someterse a los mismos tipos de explicación; 3) el postulado de causalidad; lo que interesa explicar son las causas y condiciones que producen creencias o estados de cosas; 4) el postulado de la reflexividad de las explicaciones respecto a la propia sociología del conocimiento científico. ${ }^{20}$

Como todas las sociedades humanas ticnen necesidades e intereses vitales que satisfacer, y eso influye decisivamente en las creencias que generan y aceptan, diferentes necesidades e intereses conducen a sistemas de creencias distintos e influyen también en la aparición de desacuerdos o en la construcción del consenso. Ia ciencia es nuestra forma de adaptacion a las exigencias de las estructuras naturales y sociales en que estamos inmersos y eso le da una importancia primordial a su función de predicción, manipulación y control; el conocimiento científico es una respuesta al medio mediante la observación de regularidades y la formulación de leyes," por eso admiten que en la ciencia pueden intervenir otro tipo de causas además de las sociales, como son influencias empíricas, condiciones de operatividad y de coherencia interna, etc. ${ }^{22}$ Pero estas otras catusas son, cuando menos, dirigidas y estructuradas por las primeras. De ahí que las consideren resultado de la articulación e interacción de intereses. Los intereses son el elemento más característico, y también más controvertido, del Strong Programme. Se definen como causas últimas que condicionan y estructuran las creencias, de- cisiones y evaluaciones de los cientificos.

En principio se habla de dos grandes tipos: ${ }^{23}$ los instrumentales, o legitimos', y los 'ideológicos', o secundarios. Los intereses instrumentales están relacionados con la función característica de la ciencia de predecir, manipular y controlar el medio, tanto natural, como social y cultural. Como todo conocimiento, incluido el científico, es adaptativo y está orientado fundamentalmente a la supervivencia y subsistencia, estos intereses se pueden considerar primarios y básicos. Son los que guían los distintos intereses cognoscitivos y epistémicos especializados tales como la búsqueda de leyes efectivas, la elaboración de conceptualizaciones poderosas, etc.; y también los que proporcionan a la ciencia sus caracteristicas centrales: el empirismo, la búsqueda de regularidades y la producción de rendimientos tecnológicos. Actúan como príncipios sin contenido, universales y prioritarios a la hora de producir conocimiento, aunque la forma concreta que adoptan varía de una cultura o época a otra, porque al concretarse son dirigidos y estructurados por los intereses ideologicos. ${ }^{24} \mathrm{~A}$ su vez, los intereses ideológicos son intereses sociales específicos y relativos a la organización social concreta en la que se genera el conocimiento y son secundarios no por ser menos fundamentales que los anteriores, sino porque no están explícitos, son menos básicos, y los criterios y mecanismos por los que asignan valores a creencias y a estructuras cognoscitivas están ocultos. Aunque intervienen también en la generación de creencias, su papel es más fundamental en su evaluación y aceptación, así como en las controversias y en la producción de consenso. Son intereses encubiertos dirigidos a la racionalización y persuasión, es decir, a la 
determinación social e ideológica en sentido amplio. En general, son de tres tipos:25 1) Intereses Profesionales que afectan a individuos y grupos dentro de la comunidad científica y son internos. 2) Intereses Comunitarios que afectan a la comunidad cientifica respecto a otras comunidades y al contexto cultural. 3) Intereses Sociales Generales. Estos últimos serían el tipo más representativo de intereses ideológicos y actúan en un doble sentido: a) mediante la utilización en la ciencia de patrones, modelos y actitudes culturales que funcionan en la sociedad general, y $b$ ) mediante el uso social de la naturaleza. El primer caso ocurre cuando ciertas creencias científicas y explicaciones de los fenómenos se inspiran o son influidas por concepciones sociales, políticas, etc., sastenidas en la sociedad en general. El segundo, mucho más fuerte, consiste en el uso ideológico de la naturaleza -y del conocimiento cientifico que la explicapara el control y la persuasión social. La tarea de la Sociología del Conocimiento Científico consistiría en explicar cómo estos intereses guían, configuran e incluso determinan el conocimiento científico.

\section{El Programa Relativista}

El Programa Relativista fue presentado a comienzos de los ochenta por $\mathrm{H}$. Collins y la Escucla de Bath (Pinch. Harvey, Pickering, etc.) ${ }^{26}$ como una forma de limitar los 'excesos téricos' del Strong Programme. Por eso, asume sólo dos de sus postulados - los de simetria e imparcialidad-y deja aparte los de causalidad y reflexividad. Esto lo lleva a comprometerse fuertemente con el principio de relativismo $y$, en segunda instancia, con el de constructívismo, asumiendo los restantes en un segundo plano. Su tesis central, pues, es el relativismo social tanto de las creencias como de la experiencia, que también seria socialmente construida. Respecto a la naturaleza de la sociologia del conocimiento científico como disciplina, sigue el modelo clásico de ciencia social a base de estudios empíricos de casos representativos y casos extremos, pero no pretende establecer leyes causales, ni principios generales explicativos. Este debilitamiento metodologico le exime de la necesidad de postular causas fuertes $y$, así, rechaza la tipología de los intereses del Strong Programme, porque considera que en la actividad científica juegan un papel importante distintos factores, desde el conocimiento tácito asumido por la comunidad o el grupo de investigación a los intereses cognitivos y sociales, pasando por los compromisos y el prestigio de los individuos, etc., ${ }^{27}$ los cuales sólo pueden determinarse emprricamente en cada caso. Por eso busca esas influencias sociales 'dentro' de la comunidad científica, dejando en un segundo plano los intereses sociales generales, el uso ideológico de la ciencia y el control social de la naturaleza, tan fundamentales en el caso anterior. También por esa razón, sus estudios son empíricos y descriptivos, más que interpretativos, y los lleva a cabo sobre teorías cientificas contemporáneas de las ciencias 'duras' (física de particulas, altas energías, etc.) y también casos contemporáneos de pseudo-ciencias.

Su objetivo último es demostrar la naturaleza social de la racionalidad científica y la insuficiencia de los mé. todos de control y replicación instrumental para dar cuenta de los resultados de las investigaciones de la ciencia $y$, con ello, detectar y estudiar las estructuras, factores y mecanismos sociales que subyacen ocultamente a la construcción del conocimiento científi$\operatorname{co.}^{28}$ Tales elementos se aprecian clara- 
mente cuando se producen crisis y discontinuidades en las actividades rutinarias de laboratorio o cuando, dentro de la comunidad, surgen controversias y disputas que posteriormente se resuelven por consenso. En este sentido, en lugar de intentar explicar la construcción y desarrollo de las creencias cientificas, en general se centra en tres aspectos que considera metodológicamente más interesantes: a) los métodos de experimentación y replicación cientifica y la forma en que sus resultados son determinados y construidos socialmente (los resultados experimentales pueden tener, por su propia naturaleza, distintas interpretaciones; la limitación y elirninación de esta multiplicidad se lleva a cabo mediante mecanismos de interacción social); $b$ ) las controversias y su resolución como fuentes de la aceptación del conocimiento (el acuerdo y desacuerdo científicos están regulados mediante mecanismos sociales, institucionales, retóri$\cos$, etc., que permiten el cierre de disputas favoreciendo el consenso en la comunidad); c) las ciencias marginales' (fringe sciences) para compararlas con las ciencias 'duras' (lo que permite relacionar los mecanismos de consenso y disenso científicos con el medio social más amplio, relación mucho más evidente en las ciencias marginales).

El resultado de todo esto es una desmitificación de la ciencia. En las disputas entre científicos se recurre a estrategias y recursos sociales de persuasión. Así, la explicación de cstos procesos de decisión social seria semejante a los de las acciones sociales en otras esferas. Lo que no significa que la ciencia no tenga características distintivas. Por el contrario, la principal es su método de control experimental y de 'replicación', es decir, la capacidad de repetir y reproducir experimentos. ${ }^{29}$
Se considera conocimiento objetivo lo que los científicos consideran una réplica. El problema es que entre las réplicas no hay una similaridad absoluta sino grados de semejanza. Así, el margen de error aceptable, la interpretación de los resultados y la identificación de una réplica de un experimento pueden variar y varian de hecho. Cada nuevo experimento está abierto a interpretación y, en cada nivel de decisión, es necesario tomar algunas decisiones arbitrarias desde un punto de vista cognitivo respecto a qué sea una réplica válida. Por ello, el método experimental no puede cerrar los debates. Muy al contrario, la clausura de una controversia es una decisión social que no está forzada por los hechos, sino por mecanismos de consenso social. En este sentido, el conocimiento aceptado es construido socialmente $y$, en buena medida, resultado de convenciones socialmente determinadas mediante argumentacion. ${ }^{30}$

\section{La Etnografia de la Ciencia}

Más que una corriente unitaria, es un conglomerado que incluye distintas variantes como el Programa Constructivista y los estudios de laboratorio (Knorr-Cetina, Latour, Woolgar), los análisis del discurso científico y las interpretaciones semíticas (Mulkay, Gilbert, los trabajos recientes de Woolgar), la etnometodología de la ciencia (Lynch, Livingston, etc.) e incluso la teoría de la red de actores (Callon, Latour, etc.). ${ }^{31} \mathrm{El}$ elemento común es la utilización de métodos de observación participante, interpretativos y etnográficos para eliminar cualquier sesgo teórico en el estudio de la ciencia. Puesto que la práctica cientifica y la producción de conocimiento son actividades evidentemente sociales, es necesario enfrentarse a ellas sin ninguna 
idea preconcebida describiendo las acciones e interacciones 'situadas' de los científicos como si de otra cultura se tratara. A partir de esa descripción acaba cmergiendo la forma en que la experiencia, el conocimiento e incluso la realidad, pero también los mecanismos sociales, son socialmente construidos y, al mismo tiempo, se evita la asimetría entre el sociblogo observador y el científico actor (el primero es un actor más que puede ser igualmente decodificado o está integrado en la red de actores) y se elimina la circularidad que introducen la reflexividad y el carácter 'científico' de la propia sociología. En consecuencia, insisten fundamentalmente en el principio de constructivismo $y$, sccundariamente, en el de relativismo, y se centran en el análisis microsociológico de la conducta cotidiana de los científicos, su interacción en grupos reducidos y contextos específicos (equipos de investigación, laboratorios, talk shops, etc.) y los procesos de comunicación formal e informal entre ellos.

Como resultado de todo esto, rechazan la reificación de comunidades, la existencia y relevancia de estructuras sociales subyacentes y de intereses o compromisos previamente estructurados. Todos estos elementos los disuelven en contingencias cotidianas e intereses personales que no están estructurados. La construcción de hechos y conocimientos científicos consiste precisamente en la organización social y selectiva a partir de ese desorden previo. Los 'hechos científicos' son conjuntos socialmente ordenados de datos desorganizados obtenidos artificialmente a partir de una experiencia remota y estructurados por la interacción cotidiana de los científicos en los laboratorios al mismo tiempo que se construyen, simultáneamente, la organización social y la interacción comunicativa. Por la misma razon, no tiene sentido distinguir entre factores cognitivos y sociales, ni entre influencias internas y externas, ni siquiera metodológicamente. Lo único relevante es que los científicos tienen éxito en la creación de una organización especffica y en la generación de información y esos son los procesos que deben ser descritos. Por la misma razón, prestan especial atención al lenguaje y a la comunicación entre los científicos. A fin de cuentas, si el núcleo esencial del trabajo de laboratorio consiste en la codificación ordenada y selectiva de datos de información dispersos y desorganizados, ese proceso culmina con la producción de informes científicos que construyen lingüísticamente información sistematizada, la cual está cualificada mediante modalidades. La construcción de los 'hechos' se lleva a cabo mediante la modificación o la eliminación de estas restricciones modales, lo que consigue el científico convenciendo a los demás de que sus afirmaciones tienen referencia objetiva independiente del proceso inicial de inscripción, codificación y organizacion. La argumentación y la retórica son fundamentales, en este caso, para la persuasión de los colegas y la negociación y aceptación intersubjetiva de estos 'hechos' social y lingǘsticamente construidos. En este sentido, el material sobre el que se lleva a cabo la actividad científica de construcción de hechos y conocimientos consiste básicamente en enunciados, mientras la comunicación y la argumentación lingüística son elementos centrales para la toma de decisiones y la adquisición de credibilidad. Por eso es fundamental estudiar los procesos de construcción y reificación en su contexto argumental.

Así se construyen socialmente los 'hechos' cientfficos. Lo que se entienda como tales es resultado de procesos de argumentación, comunicación e inter- 
acción social, al igual que los resultados concretos de las investigaciones experimentales y de los procesos de inscripción y registro de datos. En consecuencia, no ya el conocimiento, sino también la 'naturaleza' y la 'realidad' son construidas socialmente, y el 'mundo real' es consecuencia del trabajo de los científicos en el laboratorio más que la causa de lo que piensan. Ia actividad cientifica es una lucha por construir realidad socialmente aceptada. El resultado es que la ciencia puede entenderse como una compleja red de actores (actor-network) humanos y no humanos (pues la interacción incluye instrumentos científicos, rendimicntos técnicos, medios de comunicación, etc.) que se constituye a sí misma en interacción constante.
1. Carnap (1928; pref. a la 1." y a la $2 . " \mathrm{ed}, \mathrm{y}$ también p. 5 ss. y p. 158).

2. Reichenbach (1938: 3 ss.).

3. Reichenbach (1938: 4). Sorprendentemente, Reichenbach iniciaba su libro afimando que la epistemología, al menos en su aspecto descriptivo, formaba parte de la sociologia (ya que una de sus tareas era describir ciertos aspectos específicos del fenómeno sociologico llamado sciencian). por lo que la distincion era imprescindible para separar una de otra.

4. Reichenbach (1938:5), donde hace referencia explfcita a las 'reconstrucciones racionales' de Camap. Como en el caso anterior, esta segunda distinción era también imprescindible, ahora para separar psicología y epistemologia.

5. Que las reconstrucciones racionales diferian de la práctica real, y por tanto también podían hacerlo sus caracteristicas, era conocido y asumido por los positivistas lógicos. Así, Feigl (1969: 17) afima: aDebe tenerse en cuenta que todo esto es una reconstrucción lógica. Nunca pretendí ser una descripción del origen y desarrollo de las teorfas científicas. Sin embargo, acabo produciendose la identificación e incluso la suplantación de unas por otras.

6. Popper (1959), pref, a la edición inglesa, p. $17 y$, sobre todo, p. 31 (recuerdese que la edición alemana es de 1935).

7. Esta posicion, más tolerante, es caracteristica de la Metametodología, p. ej, Lakatos (1978; 144 5s.) con su distinción entre historia intema $y$ cxterna y su forma debilitada de entender las reconstrucciones racionales. Tambien Laudan (1977: 237) lo expone con toda claridad: *Por el contrario, si podemos mostrar que un pensador aceptó una creencia determinada, que era realmente la mejor disponible en la situación, notamos entonces que nuestra tarea explicativa ha terminado. En este modo de considerar el asunto está implícito el supuesto de que cuando un pensador hace lo que es racional hacer, no necesitamos indagar más en las causas de su acción; mientras que cuando hace lo que, de hecho, es irracional -incluso si él cree que es racionalpedimos algunas explicaciones más , y más adelante (1977: 276) continita: a Cuando, p. ej., un cientifico acepta una tradición de investigación que es mucho menos adecuada que otra rival, siempre que un cientifico sigue una teorín que es no-progresiva, cuando un cientffico concede a un problema mayor o menor importancia de la que cognoscitivamente merece, siempre que un cientifico elige entre dos tradiciones igualmente adecuadas o progresivas, en todos estos casos hemos de atender al sociologo (o al psicólogo) para su comprension, puesto que no hay posibilidad de una explicacion racional de la acción en cuestion".

8. Asi, p. ej., Merton (1973: 357) explica: *Aquf́ nos ocuparemos, de manera preliminar, de la estructura cultural de la ciencia, esto es, de un aspecto limitado de la ciencia como institución. Asf, consideraremos, no los metodos de la ciencia, sino las normas con las gue se los protege, Sin duda, los cánones metodológicos son a menudo tanto expedientes técricos como obligaciones momles, pero sólo de las segundas nos ocuparemos aquí. Este es un ensayo sobre sociología de la ciencia, no una incursión por la metodologia. Análogamente, no abondaremos aquil los hallazgos sustantivos de las ciencias (hipótesis, uniformidades, leyes) excepto en la medida en que sean atinentes a los sentimientos sociales estandarizados hacia la clencia*,

9. Un mertoniano tan conspicuo como Barber (1990: 246) Jo expone con claridad: «Los sociologos de la ciencia han enfocado su atención, pues, sobre los problemas sociológicos, la dificil tarea de relacionar una variable social o cultural o un conjunto de tales variables con otras variables sociales o culturales o conjuntos de ellas.

10. Mannheim (1929; 215) es contundente al respecto: "Se podría mostrar, en todos los casos, que no solo difieren las orientaciones fundamentales, las valoraciones y el contenido de las ideas. 
sino que la misma manera de plantear un problema, la especie de aproximación intelectual que se inicie e, incluso, las categorfas en que se ordenan las experiencias varfan de acuendo con la posición sacial del observador», De ahi los objetivos que asigna a la sociología del conocimiento: "Tienen que ser aclaradas relaciones como la siguiente: dados unos intereses determinados en una determinada coyuntura de acontecimientos, restultará un cierto tipo de pensamiento y una cierta vision del proceso social total», p. 234; «cuándo y dónde las estructuras sociales llegan a expresarse en la estructura de los juicios y en qué semialo las primeras determinan a la últimas, p. 350 ; etc.

11. Mannheim (1929:232). Que esta situación de la ciencia natural es especial lo muestra la continuación del parrafo citado: *Pero, por otra parte, es cierto que hay una amplia variedad de asuntos que sólo es accesible a ciertos sujetos, 0 en ciertos periodos historicos, y que se hace visible a traves de los propósitos sociales de los individuoss.

12. Mannheim (1929: $235 \mathrm{ss}$ ), donde va exponiendo distintas caracteristicas de la ciencia natural que no se cumplen en el resto del conocimiento con el fin de mostrar que al moderno modelo de ciencia matemática-natural no se le puede considerar como apropiado para el conocimiento en general. En otros lugares va desgranando otras razones por las cuales el contenido de la ciencia natural es independiente de factores extemos, $p$. ej.. "que no desea conocer de las co. sas más de lo que pueda ser expresado en una forma universalmente valida $y$ demostrable $y$ de no incorporarlas a la experiencia propia más alla de este punton, p. 85; aespecialmente en sus etapas cuantitativas es altamente independiente de la perspectiva historico-social del investigador, p. 379, etc.

13. El termino "caja negra" para referirse al contenido del conocimiento científico hasta entonces vedado a los estudios sociológicos o psico. logicos es de Withley (1972; 63), y fue rápidamente aceptado y utilizado por ołros sociologos como Mendelssohn, Mulkay, Gilbert, etc. Mulkay (1979) y (1980) son dos interesantes estudios de este proceso de apertura desde el punto de vista de un sociólogo. A su vez, Sánchez Navarro (1994) analiza el proceso desde un punto de vista más filosótico.

14. Un estudio detallado de estas variantes se encuentra en Sánchez Navarro y González de la Fe (1988) o Lamo de Espinosa et al. (1994: 515 ss.). Una breve exposición se encuentra al $\mathrm{co}$. mienzo del libro de Torres Albero (1994: 12-22) que, por otra parte, es un analisis muy sugerente sobre los problemas del orden, la autoridad y el cambio científico desde la sociología de la ciencia.

15. El intento más conocido de llevar a cabo esta redefinición está en Bloor (1983). Otro es la propuesta de una "ciencia del conocimiento' desarollada por Collins (1990). Interpretaciones mucho mas radicales que incluyen también la tecnologia se encuentran en Callon (1989), Law (1986. 1992). Latour (1987, 1993) o Strum y Latour (1987). Fuller, De Mey, Shinn y Woolgar (1989) incluye artículos sobre la cuestión desde distintos puntos de vista (psicologia, sociologta, filosofia). como también McMullin (1992). Incluso en intentos filosoficos recientes de recuperar estas nociones se aprecia el impacto producido por la sociologfa del conocimiento cientffico, como en Olivé (1988), Fuller (1988, 1993), Giere (1992) o Kitcher (1993). Sobre esta cuestion, véase el artfculo de A. Gómez Rodriguez en este mismo número,

16. P. ej., Woolgar (1981), Ashmore (1989), Pickering (1992) o la discusión del Programa Relativista en el número 1 del volumen 12,1982, de Social Siudies of Science.

17. La formulación inicial está en Bloor (1973 y 1976: cap. 1) y Bames (1974 y 1977), aunque en este de forma mas moderada. Shapin (1982) incluye una exhaustiva relacion de analisis historicos reivindicados en favor de la interpretación instrumental del SP. También son importantes Bames (1980, 1981a, 1981b y 1982), Bloor (1981. 1982, 1984 y el Afterword a la $2 . "$ ed. de 1976). Barnes y Shapin (1979), Bames y Bloor (1982), MacKenzie (1981 y 1990), y Shapin y Schaffer (1985). También son muy ilustrativas la polémica con Laudan en la Conferencia de Toronto recogida en Philosophy of the Social Sciences, 11.1981. 173-243; el numero especial de Studies it History and Philosophy of Science, 13 (1982), dedicado a la Sociologia del Conocimiento; las recopilaciom nes de Brown (1984) y Hronsby, Feher y Dajka (1988).

18. Bloor (1976: 3) afirma: aSus ideas estanán en el mismo lenguaje causal que las de cualquier otro cientifico. Su interés seru localizar las regularidades y principios genemles que parecen estax funcionando dentro del dominio de sus datos. Su propósito sera construir teortas que expliquen estas regularidades. Bames (1977: 85), sin embargo, es más débil y reconoce: «Es cierto que no se proponen leyes o condiciones necesarias que enjacen el conocimiento y al orden social y que no se presentan instrucciones abstractas para la investigación y explicación de cuerpos de conocimiento*, algo parecido mantiene en su (1982: 115).

19. Shapin (1982: 159). En términos mấs generales también se encuentra en Bloor (1976: 142). Bames (1977: cap. 3), etc.

20. Asf los explicita Bloor (1976: 4).

21. Bloor (1976: 17).

22. Asi lo admite Bloor (1982: 274 55.). Mucho más claro es Barres (1977: 24): *El conocimiento no puede ser entendido más que como el produc- 
to generado por hombres que operan en terminos de un interés en la predicción y el control confor: mado por lo especifico de su situacion*; por eso no llega en ningún caso a la sobresociologizacion. Asr, en su (1974: 7) dice: o Ocasionalmente, el trabajo de que disponemos da la sensación de que la realidad no tiene nada que ver con lo que es socialmente construido o negociado para que cuen. te como conocimiento natural, pero seguramente podemos asumir que esta impresion es un sub. producto accidental del analisis sociologico superentusiasta y que los sociologos en su conjunto reconocerán que el mundo restringe de alguna mancra lo que se cree que existen.

23. Bames es quien más desarrolla la teoria de los intereses. Asi, afirma: "El conocimiento crece bajo el impulso de dos grandes intereses; un interes abierto en la prediccion, manipulación y control y un interés encubierto en la racionalización y la persuasión. Nuestra definición de determinacion ideologica la ha identificado como el modo de operacion de este segundo gran interés»; Bar. nes (1977: 38). Afirmaciones semejantes se encuentrar en Barnes (1981a: 324-328); Barnes y Mackenzie (1979; 54 5s.): Shapin (1982: 139-141); Bloor (1982: 307-308), etc.

24. Las posiciones respecto a las relaciones entre ambos tipos de intereses no son unánimes: Asi, Bloor (1982, 1983: 303) las radicaliza considerandolos indistinguibles; pero la opinion mas general es que pueden separarse, al menos meto. dologicamente; Bames (1982: 208 y 218); Barnes y Mackenzie (1979: 66).

25. Un desarrollo detallado se encuentra en Shapin (1982: 164 ss.).

26. Ia exposición inicial está en collins (1981a). Tambien son importantes Collins (1981b, 1983,1985 y 1990), Collins y Pinch (1982 y 1993), Pickering (1984), Pinch (1986) o Traweek (1988).

27. Sobre los intereses cognitivos y la coherencia con el conocimiento establecido habla Pickering (1984); sobre los compromisos y el prestigio Harvey (1981), etc, En Collins (1981b: nota 17) aparece una larga lista de acciones 'políticas' que pueden influir en el curso de una disputa y que van desde presionar a los editores de revistas para que acepten o rechacen artículos en favor o en contra de una de las posiciones en conflicto hasta intentar aislar académicamente al rival o influir en la asignación de fondos económicos. También Collins (1983:276).

28. Asi, Collins (1985: 84) afirma nno sabemos si hemos construido un buen detector hasta que hemos intentado y obtenido el resultado correcto. Pero no sabemos cual es el resultado correcto hasta,.. y así ad infinitum. Lo que quiere probar- se es que xel hombre juega una parte activa en ver regularidades más que registrarlas pasiva. mente: y que no es la regularidad del mundo la que se impone a nuestros sentidos, sino la regula. ridad de nuestras creencias institucionalizadas la que se impone al mundo*, Collins (1985: 75 y 148).

29. Por eso Collins (1985; 19) considera la 'replicabilidad' como *la contrapartida científica institucionalizada de la estabilidad de la percepción y *el criterio de demarcación del conocimiento objetivon.

30. Harvey (1981: 161) llega a decir que "no tenemos acceso directo a la naturaleza. Enfrentamos la realidad a traves de las práticas experimentales en que somos socializados, prácticas que se localizan en un contexto social... Así nuestro conocímiento sobre el mundo no puede ser aislado del contexto social en el cual ese conocimiento es generados. Tambien Collins (1981a; 3) afirma: «El mundo natural tiene un papel minitho o inexistente en la construcción del conocimiento cientificon.

31. El Programa Constructivista es la propuesta de Knorr-Cetina (1981) y tambien Knorr-Cetina y Mulkay (1983), aunque esta es una compilación general que incluye también trabajos de las otras líneas etnograficas. En ambos casos, el núcleo fundamental es el analisis del trabajo en laboratorio donde la contribución fundamental es Latour y Woolgar (1979) y tambien Lynch y Woolgar (1990), aunque éste se aproxima más a los trabajos etnometodológicos. Los trabajos clasicos en Etnometodología de la Ciencia son los de Lyndh (1982 y 1984) y Livingston (1986). En cuanto a los análisis del discurso, son fundamentales Gilbert y Mulkay (1984) y Mulkay (1985). pero también es muy ilustrativo Myers (1990). En la misma linea, pero más cercano a planteamientos semióticos derivados de su preocupación por el problema de la reflexividad, están Ashmore (1989) y Woolgar (1988b). A su vez, Woolgar (1988a) es una pequetia introducción al tema en la onda de los trabajos de Woolgar. La teoría de la red de actores la desarrollan Callon (1989) y Latour (1987 y 1988) como consecuencia de su interes en introducir el analisis de la tecnologfa en el estudio social de la ciencia. En este sentido también son importantes Law (1986), Callon, Law y Rip (1987) y Bijker. Hughes y Pinch (1987), en los que se empezo a plantear ese tema. Woolgar (1991) describe este giro hacia la tecnología, al igual que Bijker y Law (1992). Finalmente, Latour (1990) es una revisión de la etnografia de la ciencia en la estela de su clásico (1981). 


\section{REFERENCLAS}

ASHMoRE, M. (1989): A Question of Reflexivity: Writing the Sociology of Sctentific Knowledye. Chicago, U. Chicago P.

Barber, B. (1990): Social Sindies of Science, New Brunswick, Transaction.

Barkes, B. (1974): Scientific Knowledge and Sociological Theory, Londres, RKP.

- (1977): Interests and the Growth of Knowledse. Londres, RKP.

- (1980): a On the Causal Explanation of Scientific Judgment:. Social Science Infomation, 19 $685-695$.

- (1981a): aOn the Conventional Character of Knowledge and Cognition», Philosophy of So cial Sciences, 11, 303-333.

- (1981b): on the 'Hows' and 'Whys' of Cultural Change», Social Studies of Science, 11, 481.498.

- (1982): Kuhn y la ciencia social, México, FCE, 1986.

- y D. McKenzie (1979): *On the Role of Interests in Scicntific Changew, en Wallis (ed), On the Mangins of Science: The Social Construction of Rejected knowledge, Keele, Sociological Review Monograph.

- y S. Shapin (eds.) (1982): Natural Order: Historical Studies of Scientific Culture, Londres Sage.

Buker, W., T. Hughes, y T. Pinch (eds.) (1987): The Social Construction of Technological Systemu, Cambridge, MA, MIT $\mathrm{P}$.

- y J. LAW (eds.) (1992): Shaping Technology Building Society, Cambridge, MA, MIT P.

BLoor, D. (1973): wittgenstein and Mannheim on the sociology of mathematicsn. Studies in History and Philosophy of Science, 4, 173-191.

- (1976): Knowledge and Social Intagery, Londres, RKP; $2 . "$ ed. en U. Chicago P.; 1991

- (1981): "The Strength of the Strong Programmes, Philosophy of the Social Sciences, $11,199-$ 213.

- (1982): Durkheim and Mauss Revisited: Classification and the Sociology of Knowledgew, Studies in History and Philosophy of Science. 13, 267-298.

- (1983): Witgenstein: A Social Theory of Knowledge, Londres, MacMillan.

- (1984): *The Sociology of Reasons: Or Why 'Epistemic Factors' are Really "Social Factors"”. en Brown (ed), Scientific Rationality: The Sociological Tun, Dordrecht, Reidel.

- y B. BARNES (1982): *Relativism, Rationality and the Sociolony of Knowledgex, en Lukes $y$ Hollis (eds.), Rationality and Relativism, $\mathrm{Ox}-$ toud, Blackwell.

Brown, J. (ed.) (1984): Scientific Racionality: The Sociological Tum, Dondrecht, Reidel.

Callón. M. (ed.) (1989): La Science et ses Réseaux París, La Découverte.
-, J. LAW, y A. Rtp (eds.) (1987): Mapping the Dynamics of Science and Technology, Londres, MacMillan.

CARnap, R. (1928): Der Logische Aufbau der Welt, Berlín, Weltkreis; 2," ed. F. Meiner, 1961.

Colluns, H. (ed.) (1981a): Knowledge and Controversy: Studies of Modem Natural Sctence, Social Studies of Science, 11.

- (1981b): "Son of Seven Sexes: The Social Destruction of a Physical Phenomenons, Social Studies of Science, 11, 33-62.

- (1983): :The Sociology of Scientific Knowledge: Studies of Contemporary Sciencen, Ammual Review of Sociology, 9, 265-285.

-(1985): Changing Order: Replication and Induction th Scientific Practice, Londres, Sage.

- (1990): Artificial Expents, Cambridge, MA, MTT P.

-, y T. PwCH (1982): Frames of Mearing, Londres, Routledge.

- y T. Pinch (1993): The Golem: What Everyone Should Know about Science, Cambridge, Cambridge U.P.

FejcL, H. (1969): "The Origin and Spirit of Logical Positivism", en Achinstein y. Barker (eds.), The Legacy of Logical Positivism, Baltimore, J. Hopkins P., 1969.

Fuluer, S. (1988): Social Epistemology, Bloomington, Indiana U.P.

- (1993): Philosophy, Rhetoric and the End of Knowledge, Madison, U. Wisconsin P.

-, M. dE Mey, T. Shinn, y S. Woulgar (ods.) (1989): The Cognitive Tum, Dordrecht, Kluwer.

Grere, R. (ed.) (1992): Cognitive Models of Science, Minneapolis, U. Minnesota $P$.

Gllbert, G. y M. Mulkay (1984): Opening Pajidora's Box, Cambridge, CUP.

Harvex, B. (1981): «Plausibility and the Evaluation of Knowledge: A Case Srudy of Experimental Quantum Mechanics, en Collins (1981a).

Hronsky, L., M. Feher, y B. Dank (eds.) (1988): Scientific Knowledge Socialized, Budapest, A. Kiado.

KITCHER, P. (1993): The Advancentent of Science, Nueva York, Oxford U.P.

KNork-Cetins, K. (1981): The Manufacture of Khowledge: An Essay on the Constructivist and Contextual Nature of Science, Oxford, Pergamon.

-, у M. Mulkay (eds.) (1983): Science Observed, Londres, Sage.

Lakatos, I, (1978): La Metodologia de los Programas de Investigación Ctentifica. Madrid. Alianza, 1983 .

Lamo de Espinosa, E., J. Gonzalez Garcla, y C. ToRRES ALBero (1994); La Sociologia del Conocimituto y de la Ciencia, Madrid, Alianza. 
Latouk, B. (1981): Nunca hemos sido Modemos, Madrid, Debate, 1993.

- (1987): Ciencia en Accion. Barcelona, Labor; 1992.

- (1988): The Pasteurization of France, Cambridge, MA, Harvard U.P.

- (1990): «Postmodern? No, Simply Amodern. Steps Towards an Anthropology of Science: An Essay Reviewn, Situdies in History and Philosophy of Science, 21, 145-171.

- (1993): La Clef de Berlin, París, La Découverte.

-, y S. Woolgar (1979); Laboratory Life. BeverIy IIIlls, Sage; 2." ed., Princeton, Princeton U.P., 1986 (trad, cast. en Alianza, 1995).

Lauban, L. (1977): El Progreso y sus Problemas, Madrid, Encuenitro, 1986.

LAw, J. (ed.) (1986): Power, Action and Belief. A New Sociolory of Knowledge?, Keele, Sociological Review Monograph.

- (ed.) (1992): A Sociology of Montsters. Essays on Power. Technology and Domination, Londres, Sociological Review Monograph.

Luvinaston, E. (1986): The Ethnomethodological Foundations of Mathenatics, Boston, RKP.

LrNch, M. (1982): aTechnical Work and Critical Inquiry", Social Studies of Science, 12, 499-534,

- (1984): Art and Artifact in Laboratory Life, Londres, RKP.

-. y S. Woolgar (eds.) (1990): Representation in Scientific Practice, Cambridge, MA, MIT P.

MaCKenże, D. (1981): Statistics iti Britain, 1865 1930: The Social Construction of Scientific Knowledge, Edimburgo, Edinburgh U.P.

- (1990): Inventig Accuracy. A Historical Sociology of Nuclear Missile Guidance System, Cambridge, MA, MIT P.

McMulum, E. (ed.) (1992): The Social Dintentsions of Science, Notre Dame, Notre Dame U.P.

MaNkHEM, K. (1929): Ideologia y Utopia, Madrid. Aguilar, 1958.

Merton, R. (1973): La Sociología de la Ciencia, Madrid, Alianza, 1977.

MuLer, M. (1979): Science and the Sociology of Knowledge, Londres, Allen \& Unwin.

- (1980): a Sociology of Science in the West., Current Sociology, 28, 1-184.

- (1985): The Word and the World, Londres, Allen \& Unwin.

Mreas, G. (1990): Writing Btology: Texts in the So- cial Construction of Scientific Knowledge, Madison, U. Wisconsin P.

OLwe, L. (1988): Conociniento, Sociedad y Reali. dad, México, FCE.

Pickering, A. (1984): Constructing Quatks, Edimburgo, Edinburgh U.P.

- (ed.) (1992): Setonce as Practice and Culture, Chicago, U. Chicago P.

PuNCH, T. (1986): Confrontüng Nature, Dordrecht, Reidel,

Popper, K. (1959): La Lógica de la Investigaciónt Cientffica, Madrid. Tecnos, 1962.

Reicuenbach, H. (1938): Experience and Predicfion, Chicago, U. Chicago $\mathrm{P}$.

SAnCHEZ NAYARRO, J. (1994): "Natumalización y Factores Sociales en la Ciencia*, en Bustos et al. (eds.), Perspectivas Actuales de Logica y Filo. sofia de la Ciencia, Madrid, Siglo XXI.

- - y T. GonzAlez de la FF. (1988): uLas Sociologias del Conocimiento Cientificon, REIS, 43, 75-124,

SilapiN, S. (1982): "History of Science and its Sociological Reconstructions*. History of Science, $20,157-211$.

-. y S. SCHAFFer (1985): Leviathan and the Air Punp, Princeton, Princeton U.P.

Strum, Si, y B. LATOUR (1987): athe Meanings of Social: From Baboons to Humans*, Soctal Sciences Information, 26, 783-802.

Torres Albero, C. (1994): Sociologia Politica de la Ciencia, Madrid, CIS.

TraWEEK, S. (1988): Beamtinies and Lifetimes: The World of High Energy Physics, Cambridge, MA, Harvard U.P.

Whrtey, R. (1972): «Black boxism and the sociology of science: A discussion of the majox developments in the field". Sociological Review Monographs, 18, 61-92.

WOOLGAR, S. (1981): *Interests and Explanation in the Social Study of Sciencen, Social Studies of Science, 11, 365-394.

- (1988a): Ciencia: Abriendo la Caja Negra, Ba1celona, Anthropos, 1991.

- (ed.) (1988b): Knowledge and Reflexivity. Londres, Sage.

- (1991): "The Tum to Technology in Social Studies of Science», Techrology \& Human Values, $16,20-50$. 